\title{
Clinical Usefulness of the HFA-PEFF Diagnostic Scoring System in Identifying Late Elderly Heart Failure With Preserved Ejection Fraction Patients
}

\author{
Yoshihiro Seo, MD, PhD; Tomoko Ishizu, MD, PhD; Masaki Ieda, MD, PhD; \\ Nobuyuki Ohte, MD, PhD for the J-LONG Study Investigators
}

\begin{abstract}
Background: Diagnosis of heart failure with preserved ejection fraction (HFpEF) remains challenging in elderly. This study investigated the diagnostic ability of the HFA-PEFF scoring system in elderly patients ( $>75$ years of age).

Methods and Results: This study enrolled 286 subjects aged $>75$ years (130 men; mean $[ \pm \mathrm{SD}]$ age $81.5 \pm 5.1$ years): 95 healthy controls, 98 with hypertension (HT), and 93 with HFpEF. The HFA-PEFF score was calculated as a sum of points in functional, morphological, and biomarker domains. In the HFpEF group, $84 \%, 84 \%$, and $70 \%$ of subjects met the major functional, morphological, and biomarker criteria for HFpEF, respectively. Thus, 73 subjects with $\mathrm{HFpEF}(78 \%)$ were diagnosed as having HFpEF using the HFA-PEFF scoring system. In contrast, among the healthy controls and subjects with $\mathrm{HT}, 52 \%$ and $72 \%$, respectively, met the major functional criteria for $\mathrm{HFpEF}, 28 \%$ and $53 \%$, respectively, met the morphological criteria, and $0 \%$ and $24 \%$, respectively, met the biomarker criteria. As such, 32 subjects with $\mathrm{HT}(33 \%)$ were diagnosed with $\mathrm{HFpEF}$. Even in the healthy control group, $72 \%$ were classified as having an intermediate probability of $\mathrm{HFpEF}$, and 3 were diagnosed with $\mathrm{HFpEF}$.
\end{abstract}

Conclusions: In the late elderly, the HFA-PEFF scoring system diagnosed subjects with HFpEF precisely. In addition, this scoring system may be able to detect early stage HFpEF in the subclinical population.

Key Words: Diastolic function; Echocardiography; Elderly; Heart failure with preserved ejection fraction (HFpEF); Natriuretic peptide

$\mathbf{J}$ apan, Italy, and Germany already have a super-aged society, and it is projected that there will be a global increase in the proportion of the aged population, primarily the late elderly (i.e., those aged $\geq 75$ years). ${ }^{1,2}$ In the elderly, heart failure (HF) with preserved ejection fraction (HFpEF) is the major phenotype of HF. ${ }^{3-5}$ Therefore, reducing the incidence and slowing the progress of HFpEF, as well as preventing death from this disease, have become social issues. However, awareness of HFpEF is low, even among healthcare professionals. ${ }^{4}$ Diagnosis of HFpEF in elderly patients with comorbidities and no obvious signs of central fluid overload is challenging, particularly in the late elderly. Left ventricular (LV) diastolic dysfunction is the main pathophysiology in HFpEF, but is not essential in HFpEF. ${ }^{5-10} \mathrm{HFpEF}$ is a clinical syndrome, and its heterogeneity may make diagnosis difficult. ${ }^{11}$ Recently, the Heart Failure Association (HFA) of the European Society of Cardiology proposed a new algorithm, the HFA-PEFF score, to identify HFpEF based on a comprehensive diagnostic workup using the HFA-PEFF scoring system. ${ }^{12,13}$ Because in the real world HFpEF is more common in the late elderly, the value of the HFA-PEFF scoring system has to be validated in this population.

The Japanese eLderly data Of Normal echocardioGraphy (J-LONG) study was designed to establish normal value for echocardiographic parameters in the late elderly (i.e., those aged $>75$ years) because of a lack of such values in this population. ${ }^{14}$ In addition to healthy controls, the J-LONG study also enrolled patients with hypertension (HT) and those with HFpEF. The aim of the present study, using data from the J-LONG study, was to investigate the diagnostic ability of the HFA-PEFF scoring system in patients with HFpEF aged $>75$ years.

\section{Study Population}

Participants were enrolled in the J-Long study between April 2017 and March 2019. In all, 402 subjects aged $>75$ years were enrolled, including 130 healthy controls, 115 with HT, 93 with HFpEF, and 64 with aortic stenosis. HT was defined as an office blood pressure of at least $140 \mathrm{mmHg}$

Received July 19, 2020; revised manuscript received September 3, 2020; accepted September 23, 2020; J-STAGE Advance Publication released online November 28, 2020 Time for primary review: 30 days

Department of Cardiology, Nagoya City University Graduate School of Medical Sciences, Nagoya (Y.S., N.O.); Department of Cardiology, Faculty of Medicine, University of Tsukuba, Tsukuba (T.I., M.I.), Japan

Mailing address: Yoshihiro Seo, MD, PhD, Department of Cardiology, Nagoya City University Graduate School of Medical Sciences, 1 Kawasumi, Mizuho-cho, Mizuho-ku, Nagoya 467-8601, Japan. E-mail: yo-seo@med.nagoya-cu.ac.jp

All rights are reserved to the Japanese Circulation Society. For permissions, please e-mail: cj@j-circ.or.jp

ISSN-1346-9843 
systolic or $90 \mathrm{mmHg}$ diastolic, a home blood pressure of at least $135 \mathrm{mmHg}$ systolic or $85 \mathrm{mmHg}$ diastolic in untreated cases, or currently receiving antihypertensive medication. Subjects with HFpEF were defined as those with a history of hospitalization for HF, those currently undergoing $\mathrm{HF}$ treatment, and those with a left ventricular ejection fraction (LVEF) of $\geq 50 \%$ at the time of registration in whom there were no underlying diseases of $\mathrm{HF}$, such as hypertrophic cardiomyopathy, amyloidosis, sarcoidosis, constrictive pericarditis, advanced mitral valve disease, advanced aortic valve disease, and pulmonary arterial and chronic thromboembolic pulmonary HT. After excluding subjects with aortic stenosis, participants with complete datasets to enable assessment of the HFA-PEFF score were screened for inclusion in this study. Finally, 286 subjects (130 men; mean $[ \pm \mathrm{SD}]$ age $81.5 \pm 5.1$ years, range $75-98$ years) were enrolled in this study: 95 healthy controls, 98 with HT, and 93 with HFpEF. The main reason for exclusion from the study was the lack of B-type natriuretic peptide (BNP) data.

This study investigation conformed to the principles outlined in the Declaration of Helsinki. The study was approved by the Ethics Committee of the University of Tsukuba (Reference no. H28-119), as well as the local ethics committee of each participating institute. Informed consent was obtained from all subjects before they were included in the study.

\section{Echocardiographic Data}

All images were reviewed offline by the headquarter using vendor-independent 2D Cardiac Performance Analysis software (TomTec Imaging System, Munich, Germany).

Comprehensive echocardiographic studies were performed according to established guidelines. ${ }^{15,16} \mathrm{LV}$ enddiastolic diameter (LVDd), interventricular septum thickness (IVSTd), posterior wall thickness (PWTd), and LV endsystolic diameter (LVDs) were measured in parasternal long-axis views. LV volumes and LVEF were measured by the disk summation method from the apical 4- and 2-chamber views. Relative wall thickness was calculated as $(2 \times$ PWTd/LVDd). LV mass (LVM) was calculated using Devereux's formula. Maximum left atrial volume (LAV) was measured by the disk summation method from the apical 4- and 2-chamber views. LVM and LAV were corrected for body surface area (LVMI and LAVI, respectively). The tissue Doppler-derived early diastolic mitral annular velocity (e') was measured at septal and lateral wall sites in the apical 4-chamber view. The ratio of early diastolic mitral inflow velocity (E) to e' (E/e') was calculated as the mean of septal and lateral E/e'. Tricuspid regurgitation peak velocity (TRV) was derived from peak tricuspid regurgitation jet velocity.

Speckle tracking echocardiography (STE) was performed using the TomTec Imaging System. In an end-systolic frame, a region of interest was traced on the endocardial cavity interface using a point-and-click approach. The region of interest was automatically selected to approximate the myocardium between the endocardium and epicardium. The region of interest was adjusted further to ensure that all myocardial regions were included. Then, the software captured the myocardium, automatically tracking its motion and thickening in subsequent frames. Finally, the myocardium was divided into 6 segments in each plane. For assessment of LV global longitudinal strain (GLS), the mean of the longitudinal systolic negative peak values obtained from the 18 LV segments in the apical 4-, 3-, and 2-chamber views was calculated. Segments were discarded if tracking was of poor quality.

\section{Calculation of the HFA-PEFF Score}

The HFA-PEFF score was calculated using the proposed algorithm, ${ }^{12}$ which assigns 2 points for a major criterion and 1 point for a minor criterion within each functional, morphological, and biomarker domain (Supplementary Figure). A total score of 5 or 6 is considered to be diagnostic of HFpEF, whereas scores of 0 or 1 are considered to indicate that a diagnosis of HFpEF is very unlikely. Patients with scores in the range $2-4$ are considered as being at intermediate probability of having $\mathrm{HFpEF}$; specifically, HFpEF could not be diagnosed in these patients using the HFA-PEFF scoring system.

As a diagnostic control for HFpEF to examine the added value of a comprehensive evaluation using the HFA-PEFF score, the Doppler echocardiographic algorithm for the evaluation of LV diastolic function of the American Society of Echocardiography (ASE) and the European Association of Cardiovascular Imaging (EACVI) was used. ${ }^{16}$

\section{Statistical Analysis}

Variables were compared between groups using 1-way analysis of variance (ANOVA) with a post hoc TukeyKramer test. Student's t-tests were used to compare continuous variables between 2 groups, whereas $\chi^{2}$ tests were used to compare categorical variables between groups. Statistical analyses were performed using SPSS ver. 25 (SPSS Inc., Chicago, IL, USA).

\section{Results}

\section{Clinical Characteristics}

The clinical characteristics of the healthy control, HT, and HFpEF groups are summarized in Table 1. Age and sex distributions did not differ among groups. Systolic blood pressure was higher in the HT than HFpEF group, and diastolic blood pressure was lower in the HFpEF than other groups. Atrial fibrillation (AF) was observed in approximately one-quarter of subjects in the HFpEF group. Hemoglobin, albumin, and estimated glomerular filtration rate (eGFR) were lower in the HFpEF than other groups.

\section{Comparisons of Echocardiographic Parameters and BNP Concentrations}

As indicated in Table 2, both LVDd and LVDs were larger in the HFpEF than other groups. In addition, IVSTd and PWTd were greater in the HFpEF than healthy control group. LVEF was lowest in the HFpEF than in the other 2 groups.

Of the functional parameters contributing to the HFAPEFF score, e' at the septum was smaller in the HFpEF than healthy control group but did not differ from that in the HT group; there was no significant difference in e' at the lateral wall among the 3 groups. E/e' and TRV were significantly higher in the HFpEF than other groups. Adequate speckle tracking to obtain GLS was confirmed in $5,066(98.6 \%)$ segments, with significant differences in GLS among the 3 groups. Of the morphological parameters, although there were no differences in LAVI in subjects with AF between the HT and HFpEF groups, among subjects with sinus rhythm, LAVI was significantly larger in the HFpEF than other groups. Similar results were observed for LVMI, although relative wall thickness did 


\begin{tabular}{|c|c|c|c|c|}
\hline & $\begin{array}{l}\text { Healthy control } \\
\qquad(n=95)\end{array}$ & $\begin{array}{l}\text { Hypertension } \\
(n=98)\end{array}$ & $\begin{array}{l}\text { HFpEF } \\
(n=93)\end{array}$ & $P$ value \\
\hline Age (years) & $79.4 \pm 4.4$ & $80.5 \pm 3.9$ & $80.5 \pm 3.2$ & 0.08 \\
\hline Male sex & $42(44)$ & $44(45)$ & $44(47)$ & 0.90 \\
\hline $\mathrm{BSA}\left(\mathrm{m}^{2}\right)$ & $1.52 \pm 0.15$ & $1.53 \pm 0.18$ & $1.49 \pm 0.17$ & 0.40 \\
\hline $\mathrm{BMI}\left(\mathrm{kg} / \mathrm{m}^{2}\right)$ & $23.3 \pm 2.9$ & $23.3 \pm 3.3$ & $22.4 \pm 4.1$ & 0.13 \\
\hline $\mathrm{SBP}(\mathrm{mmHg})$ & $129 \pm 14$ & $137 \pm 16^{\dagger}$ & $127 \pm 21$ & 0.002 \\
\hline $\mathrm{DBP}(\mathrm{mmHg}$ & $71 \pm 10$ & $71 \pm 12$ & $66 \pm 14^{*}$ & 0.006 \\
\hline HR (beats/min) & $70 \pm 11$ & $70 \pm 12$ & $66 \pm 12$ & 0.056 \\
\hline Hypertension & 0 & $98(100)$ & $76(82)$ & $<0.001$ \\
\hline Diabetes & 0 & $24(25)$ & $27(29)$ & $<0.001$ \\
\hline $\mathrm{AF}$ & 0 & $7(7.1)$ & $24(26)$ & $<0.001$ \\
\hline Hemoglobin (g/dL) & $13.1 \pm 1.3$ & $12.9 \pm 1.5$ & $11.5 \pm 1.8^{*}$ & $<0.001$ \\
\hline Albumin (g/dL) & $4.1 \pm 0.3$ & $4.1 \pm 0.4$ & $3.6 \pm 0.6^{\star}$ & $<0.001$ \\
\hline eGFR $\left(\mathrm{mL} / \mathrm{min} / 1.73 \mathrm{~m}^{2}\right)$ & $59.3 \pm 15.5$ & $55.1 \pm 16.1$ & $45.5 \pm 16.9^{*}$ & $<0.001$ \\
\hline
\end{tabular}

Unless indicated otherwise, data are given as the mean $\pm S D$ or $n(\%) .{ }^{*} P<0.001$ compared with the other groups; ${ }^{\dagger} \mathrm{P}<0.01$ compared with the HFpEF group. AF, atrial fibrillation; BMI, body mass index; BSA, body surface area; DBP, diastolic blood pressure; eGFR, estimated glomerular filtration rate; HFpEF, heart failure with preserved ejection fraction; HR, heart rate; SBP, systolic blood pressure.

\begin{tabular}{|c|c|c|c|c|}
\hline & $\begin{array}{l}\text { Healthy control } \\
\quad(n=95)\end{array}$ & $\begin{array}{l}\text { Hypertension } \\
\qquad(n=98)\end{array}$ & $\begin{array}{l}\text { HFpEF } \\
(n=93)\end{array}$ & $P$ value \\
\hline \multicolumn{5}{|c|}{ Echocardiographic parameters } \\
\hline LVDd (mm) & $42.5 \pm 5.1$ & $44.3 \pm 5.2$ & $46.3 \pm 6.2^{\ddagger \ddagger . \dagger}$ & $<0.001$ \\
\hline LVDs (mm) & $26.2 \pm 3.8$ & $27.3 \pm 3.8$ & $29.6 \pm 6.3^{\ddagger \ddagger}, \dagger$ & $<0.001$ \\
\hline IVSTd (mm) & $9.0 \pm 1.3$ & $9.3 \pm 1.6$ & $9.8 \pm 2.1^{\ddagger}$ & 0.004 \\
\hline PWTd (mm) & $8.9 \pm 1.2$ & $9.4 \pm 1.6$ & $9.7 \pm 1.8^{\ddagger}$ & 0.002 \\
\hline LVEF $(\%)$ & $67.8 \pm 5.8$ & $66.0 \pm 6.4$ & $63.3 \pm 8.1^{\neq \neq, \dagger}$ & $<0.001$ \\
\hline $\mathrm{E}(\mathrm{cm} / \mathrm{s})$ & $59.8 \pm 13.2$ & $64.7 \pm 20.2$ & $82.5 \pm 27.6^{*}$ & $<0.001$ \\
\hline $\mathrm{E} / \mathrm{A}$ & $0.7 \pm 0.2$ & $0.7 \pm 0.2(n=90)$ & $1.0 \pm 0.5^{\star}(n=67)$ & $<0.001$ \\
\hline \multicolumn{5}{|l|}{$e^{\prime}(\mathrm{cm} / \mathrm{s})$} \\
\hline At the septum & $5.7 \pm 1.4$ & $5.3 \pm 1.5$ & $4.9 \pm 1.8^{\ddagger}$ & 0.002 \\
\hline At the lateral wall & $7.4 \pm 2.1$ & $7.0 \pm 2.0$ & $6.8 \pm 2.6$ & 0.15 \\
\hline$E / e^{\prime}$ & $9.6 \pm 2.7$ & $10.7 \pm 3.1$ & $15.8 \pm 8.2^{*}$ & $<0.001$ \\
\hline $\operatorname{TRV}(\mathrm{m} / \mathrm{s})$ & $2.3 \pm 0.3(n=82)$ & $2.4 \pm 0.4(n=82)$ & $2.6 \pm 0.5^{*}(n=88)$ & $<0.001$ \\
\hline GLS (\%) & $21.0 \pm 3.2$ & $18.9 \pm 3.1 \neq$ & $14.5 \pm 3.9^{*}$ & $<0.001$ \\
\hline \multicolumn{5}{|l|}{ LAVI $\left(\mathrm{mL} / \mathrm{m}^{2}\right)$} \\
\hline Sinus rhythm & $31.8 \pm 13.0$ & $36.1 \pm 14.2$ & $49.2 \pm 17.0^{*}$ & $<0.001$ \\
\hline $\mathrm{AF}$ & - & $54.7 \pm 21.5$ & $61.4 \pm 24.7$ & 0.52 \\
\hline \multicolumn{5}{|l|}{ LVMI $\left(g / m^{2}\right)$} \\
\hline Males & $81.3 \pm 15.9$ & $95.9 \pm 19.8^{\ddagger}$ & $110.6 \pm 39.1^{\ddagger \neq, \dagger}$ & $<0.001$ \\
\hline Female & $79.4 \pm 18.6$ & $86.4 \pm 21.6$ & $104.0 \pm 33.5^{\star}$ & $<0.001$ \\
\hline RWT & $0.43 \pm 0.08$ & $0.43 \pm 0.10$ & $0.43 \pm 0.10$ & 0.94 \\
\hline \multicolumn{5}{|l|}{ BNP (pg/mL) } \\
\hline Sinus rhythm & $15.8[22.0,30.5]$ & $41.2[25.0,79.5]^{\ddagger \neq}$ & $146.7[87.6,294.6]^{*}$ & $<0.001$ \\
\hline AF & - & $140.2[67.1,223.0]$ & $191.5[117.0,400.9]$ & $<0.001$ \\
\hline
\end{tabular}

Unless indicated otherwise, data are given as the mean \pm SD or median [interquartile range]. ${ }^{*} \mathrm{P}<0.001$ compared with all other groups; ${ }^{\ddagger} \mathrm{P}<0.01$, ${ }^{\ddagger} \neq \mathrm{P}<0.001$ compared with the healthy control; ${ }^{\dagger} \mathrm{P}<0.05$ compared with the hypertensive group. AF, atrial fibrillation; BNP, B-type natriuretic peptide; $E$, early diastolic mitral inflow velocity; $E / A$, the ratio of $E$ to $A$; e', early diastolic velocity of mitral annuls; $E / e^{\prime}$, the average of ratio of $E$ to septal e' and ratio of $E$ to lateral e'; HFpEF, heart failure with preserved ejection fraction; GLS, global longitudinal strain; IVSTd, intraventricular septal thickness at end-diastole; LAVI, indexed left atrial volume; LVDd, left ventricular (LV) end-diastolic dimension; LVDs, LV end-systolic dimension; LVEF, LV ejection fraction; LVMI, indexed LV mass; PWTd, posterior wall thickness at end-diastole; RWT, relative wall thickness; TRV, tricuspid regurgitation peak velocity. 


\section{Functional Domain}
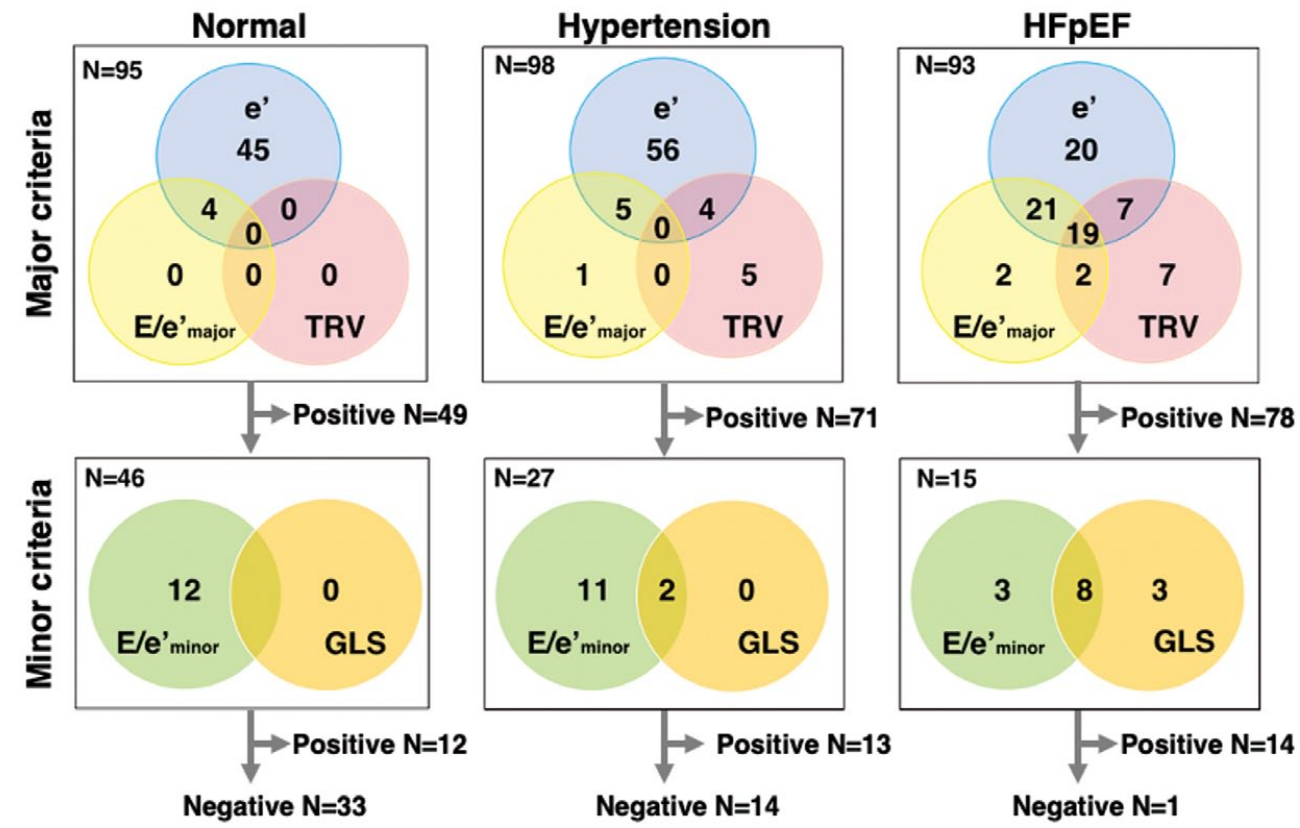

\section{Morphological Domain}
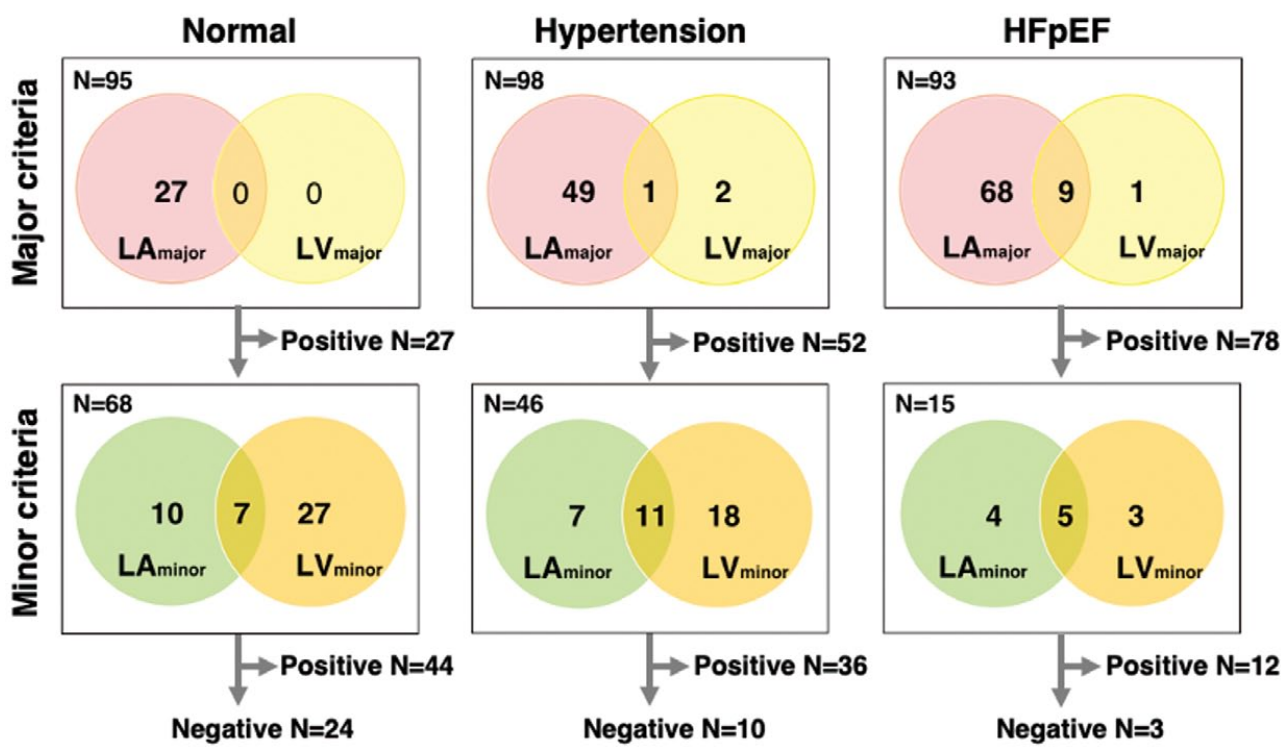

Figure 1. Venn diagrams showing the number of patients who met each major and minor criterion in the functional and morphological domains of the HFA-PEFF scoring system among the 95 healthy controls (normal), 98 hypertensive subjects, and 93 subjects with heart failure with preserved ejection fraction (HFpEF). E/e', ratio of early (E) diastolic mitral inflow velocity to velocity of mitral annuls (e'); GLS, left ventricular (LV) global longitudinal strain; LAmajor, major criterion of left atrial volume index (LAVI); LAminor, minor criterion of LAVI; LVmajor, major criterion of LV remodeling; LVminor, minor criterion of LV remodeling; TRV, tricuspid regurgitation peak velocity.

not differ between the 3 groups. There were significant differences in BNP concentrations among the 3 groups for subjects in sinus rhythm and for those with AF.

\section{Positive Rate of HFA-PEFF Score Components}

Venn diagrams in Figure $\mathbf{1}$ and Table $\mathbf{3}$ summarize the num- ber of subjects who met each criterion in the HFA-PEFF scoring system. In the functional domain, subjects meeting (vs. not meeting) the e' criterion were more common in each group. In contrast, a greater proportion of subjects in the HFpEF than other groups met the E/e' major criterion and the TRV criterion. There were significant differences 
Table 3. Subjects in the Healthy Control, Hypertensive, and HFpEF Groups Positive for Components of the HFA-PEFF Scoring System

\begin{tabular}{|c|c|c|c|c|}
\hline & $\begin{array}{l}\text { Healthy control } \\
\quad(n=95)\end{array}$ & $\begin{array}{l}\text { Hypertension } \\
\quad(n=98)\end{array}$ & $\begin{array}{l}\text { HFpEF } \\
(n=93)\end{array}$ & $P$ value \\
\hline \multicolumn{5}{|l|}{ Functional domain } \\
\hline$e^{\prime}$ at septum $<7 \mathrm{~cm} / \mathrm{s}$ & $78(82)$ & $84(86)$ & $81(87)$ & 0.61 \\
\hline$e^{\prime}$ at lateral wall $<10 \mathrm{~cm} / \mathrm{s}$ & $83(87)$ & $86(88)$ & $78(84)$ & 0.69 \\
\hline$e^{\prime}$ at septum $<7 \mathrm{~cm} / \mathrm{s}$ or e' at lateral wall $<10 \mathrm{~cm} / \mathrm{s}$ & $89(94)$ & $94(96)$ & $85(91)$ & 0.44 \\
\hline$e^{\prime}$ at septum $<5 \mathrm{~cm} / \mathrm{s}$ & $25(26)$ & $45(46)$ & $52(56)$ & $<0.001$ \\
\hline e' at lateral wall $<7 \mathrm{~cm} / \mathrm{s}$ & $38(40)$ & $55(56)$ & $54(58)$ & 0.02 \\
\hline \multicolumn{5}{|l|}{ Major criteria } \\
\hline $\begin{array}{l}\text { e' at septum }<5 \mathrm{~cm} / \mathrm{s} \text { or e' at lateral wall }<7 \mathrm{~cm} / \mathrm{s} \\
\text { (for patients } \geq 75 \text { years) }\end{array}$ & $49(52)$ & $65(66)$ & $67(72)$ & 0.01 \\
\hline$E / e^{\prime} \geq 15$ & $4(4.2)$ & $6(6.1)$ & $44(47)$ & $<0.001$ \\
\hline $\mathrm{TRV}>2.8 \mathrm{~m} / \mathrm{s}$ & 0 & $9(9.2)$ & $35(38)$ & $<0.001$ \\
\hline Subjects meeting major criteria & $49(52)$ & $71(72)$ & $78(84)$ & $<0.001$ \\
\hline Minor criteria $(n=88)^{*}$ & $\mathrm{n}=46$ & $\mathrm{n}=27$ & $\mathrm{n}=15$ & \\
\hline E/e' 9-14 & $12(26)$ & $13(48)$ & $11(73)$ & 0.004 \\
\hline GLS (absolute value) $<16 \%$ & 0 & $2(7.4)$ & $11(73)$ & $<0.001$ \\
\hline Subjects meeting minor criteria & $12(26)$ & $13(48)$ & $14(93)$ & $<0.001$ \\
\hline \multicolumn{5}{|l|}{ Morphological domain } \\
\hline LVMI $\geq 149 \mathrm{~g} / \mathrm{m}^{2}$ in men, $\geq 122 \mathrm{~g} / \mathrm{m}^{2}$ in women & $1(1.0)$ & $3(3.1)$ & $17(19)$ & $<0.001$ \\
\hline RWT $>0.42$ & $44(46)$ & $47(48)$ & $40(44)$ & 0.83 \\
\hline \multicolumn{5}{|l|}{ Major criteria } \\
\hline $\mathrm{LAVI}>34 \mathrm{~mL} / \mathrm{m}^{2}$ in sinus rhythm, $>40 \mathrm{~mL} / \mathrm{m}^{2}$ in AF & $27(28)$ & $50(51)$ & $77(83)$ & $<0.001$ \\
\hline RWT $>0.42$ and LVMI $\geq 149 \mathrm{~g} / \mathrm{m}^{2}$ in men or $\geq 122 \mathrm{~g} / \mathrm{m}^{2}$ in women & 0 & $3(3.1)$ & $10(11)$ & 0.001 \\
\hline Subjects meeting the major criteria & $27(28)$ & $52(53)$ & $78(84)$ & $<0.001$ \\
\hline Minor criteria $(n=129)^{*}$ & $\mathrm{n}=68$ & $\mathrm{n}=46$ & $n=15$ & \\
\hline RWT $>0.42$ & $32(47)$ & $27(59)$ & $5(33)$ & 0.19 \\
\hline LAVI $29-34 \mathrm{~mL} / \mathrm{m}^{2}$ in sinus rhythm, $34-44 \mathrm{~mL} / \mathrm{m}^{2}$ in AF & $17(25)$ & $18(39)$ & $9(60)$ & 0.02 \\
\hline LVMI $\geq 115 \mathrm{~g} / \mathrm{m}^{2}$ in men, $\geq 95 \mathrm{~g} / \mathrm{m}^{2}$ in women & $6(8.8)$ & $9(20)$ & $5(33)$ & 0.04 \\
\hline Wall thickness $\geq 12 \mathrm{~mm}$ & 0 & $5(11)$ & $1(6.7)$ & 0.02 \\
\hline Subjects meeting the minor criteria & $44(65)$ & $36(78)$ & $12(80)$ & 0.21 \\
\hline \multicolumn{5}{|l|}{ Biomarker domain } \\
\hline Major criterion & $\mathrm{n}=95$ & $\mathrm{n}=98$ & $\mathrm{n}=93$ & \\
\hline BNP $>80 \mathrm{pg} / \mathrm{mL}$ in sinus rhythm, $>240 \mathrm{pg} / \mathrm{mL}$ in AF & 0 & $23(24)$ & $65(70)$ & $<0.001$ \\
\hline Minor criterion $(n=198)^{*}$ & $\mathrm{n}=95$ & $\mathrm{n}=75$ & $n=28$ & \\
\hline BNP $35-80 \mathrm{pg} / \mathrm{mL}$ in sinus rhythm, $105-240 \mathrm{pg} / \mathrm{mL}$ in AF & $20(21)$ & $36(48)$ & $20(71)$ & $<0.001$ \\
\hline
\end{tabular}

Data are given as the number of subjects with percentages in parentheses. *Subjects meeting the major criterion were excluded. Abbreviations as in Table 2.

in the prevalence of subjects who met each major criterion, although the number of subjects who met the major criteria was primarily dependent on the e' criterion, with more than half of the healthy control group even meeting this criterion (Figure 1). In contrast, with regard to minor criteria, the positive rate for meting the GLS criterion was more specific for HFpEF than the minor criterion of E/e' (Figure 1). However, the addition of the GLS criterion changed the diagnosis from intermediate probability to $\mathrm{HFpEF}$ in only 2 cases.

In the morphological domain, the major criterion of LAVI differentiated the 3 groups well. In contrast, few subjects met the major criterion of LV concentric hypertrophy, even in the HFpEF group. Among the remaining subjects, those meeting the minor criteria were more common in the 3 groups with no significant differences in prevalence among the groups.

In the biomarker domain, both major and minor criteria differentiated the 3 groups well.

Figure 2 summarizes the distribution of HFA-PEFF scores in each group. In the HFpEF group, 73 subjects $(78 \%)$ had a total score of 5 or 6 , which was diagnostic of HFpEF. In contrast, in the healthy control group, only 3 subjects $(3.2 \%)$ were diagnosed with HFpEF. In the HT group, 32 subjects $(33 \%)$ were diagnosed with HFpEF. In both the healthy control and HT groups, there were fewer subjects with a score of 0 or 1 , considered to indicate that a diagnosis of $\mathrm{HFpEF}$ was very unlikely, and most subjects in these groups were classified as being of intermediate probability, with scores ranging between 2 and 4 .

Reclassification of Subjects According to the HFA-PEFF Score Figure 3 shows the reclassification of subjects in different LV diastolic dysfunction groups, determined with the ASE/EACVI algorithm, according to HFA-PEFF scores. In the HFpEF group, 24 of 26 patients $(92 \%)$ in the ASE/ EACVI intermediate probability group were reclassified as "HFpEF diagnosed" based on HFA-PEFF scores, with no subjects reclassified as "HFpEF very unlikely". In the healthy control group, 43 of 67 subjects $(64 \%)$ with normal 
LV diastolic function according to the ASE/EACVI algorithm were reclassified into the intermediate probability group based on HFA-PEFF scores. In the HT group, 42 of 45 patients $(93 \%)$ with normal LV diastolic function according to the ASE/EACVI algorithm were reclassified into the intermediate probability or HFpEF diagnosed groups using HFA-PEFF scores.

\section{Discussion}

This study investigated the ability of the HFA-PEFF score to diagnose HFpEF in the late elderly through comparisons with healthy control subjects and subjects with HT. The major findings of this study are that: (1) the proportion of subjects meeting the HFA-PEFF criteria differed significantly among the 3 groups, although some criteria showed a relatively high rate of conformable cases even in the normal and HT groups; (2) the HFA-PEFF score diagnosed HFpEF subjects as HFpEF with high probability; (3) one-third of subjects with HT were diagnosed with HFpEF regardless of the presence of LV diastolic dysfunction; and (4) two-thirds of healthy control subjects and subjects HT were classified as being of "intermediate probability" rather than "very unlikely" to have HFpEF.

\section{Characteristics of Late Elderly HFpEF Subjects}

Among HFpEF subjects in this study, the prevalence of AF was higher, there was a tendency for lower heart rate, hemoglobin, eGFR, and GLS were lower, LV diastolic dysfunction was prominent, there was more LA dilatation and LV hypertrophic remodeling, and plasma BNP concentrations were higher than in the other groups. These characteristics are consistent with the clinical features of HFpEF reported previously, and the HFpEF subjects enrolled in this study were considered to be a typical HFpEF population, even though they were restricted to the elderly aged $\geq 75$ years. $., 5,10$

\section{HFA-PEFF Score Components in Elderly HFpEF Subjects}

Because myocardial relaxation abnormality is essential for LV diastolic dysfunction, ${ }^{68} \mathrm{e}$ is used as a major component in the HFA-PEFF scoring system. ${ }^{12,17}$ However, there was no significant difference in the prevalence of subjects

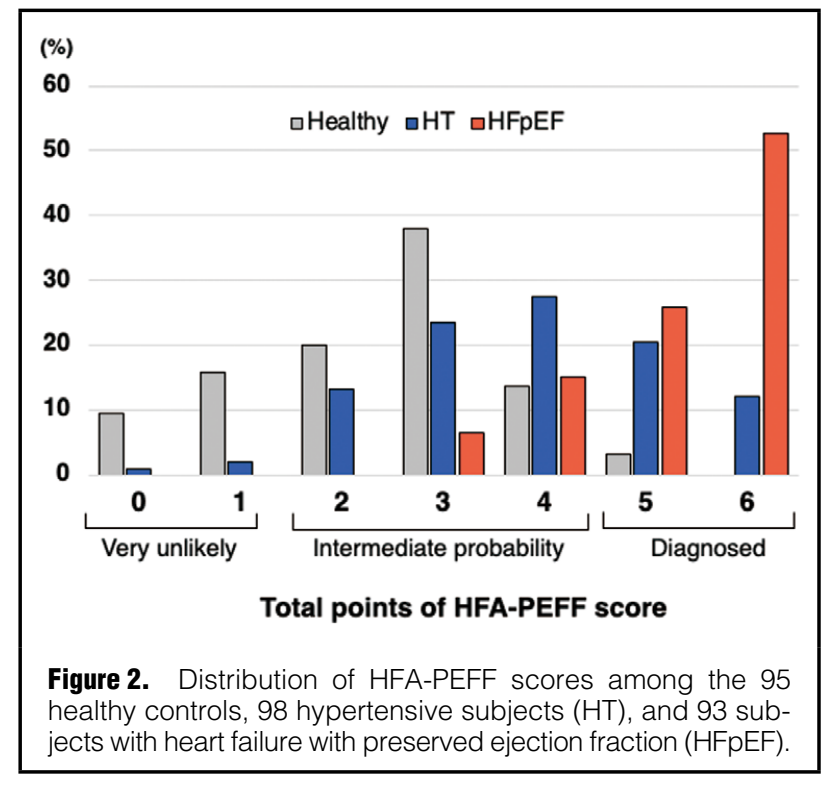

who met the general criterion of e' in the algorithm of LV diastolic functional evaluation (e' at the septum $<7 \mathrm{~cm} / \mathrm{s}$ or $e^{\prime}$ at the lateral wall $<10 \mathrm{~cm} / \mathrm{s}$ ), indicating that this level of myocardial relaxation abnormality is common even in the subclinical late elderly. Therefore, the stricter criteria for the elderly aged $\geq 75$ years in the HFA-PEFF scoring system (i.e., e' at the septum $<5 \mathrm{~cm} / \mathrm{s}$ or e' at the lateral wall $<7 \mathrm{~cm} / \mathrm{s}$ ) helped distinguish HFpEF from healthy control and HT subjects. However, the fact that more than half of all subjects (even in the healthy control group) still met the e' criterion is a possible limitation using the tissue Doppler method to evaluate myocardial diastolic dysfunction. Rather, parameters strongly suggesting elevated LV filling pressure, including high E/e', TRV, and LAVI, were more specific for HFpEF. 16

Using GLS as a parameter is a feature of the HFAPEFF scoring system and is the first time GLS has been used in an algorithm for the diagnosis of HFpEF. GLS has been used to evaluate myocardial dysfunction in subclinical

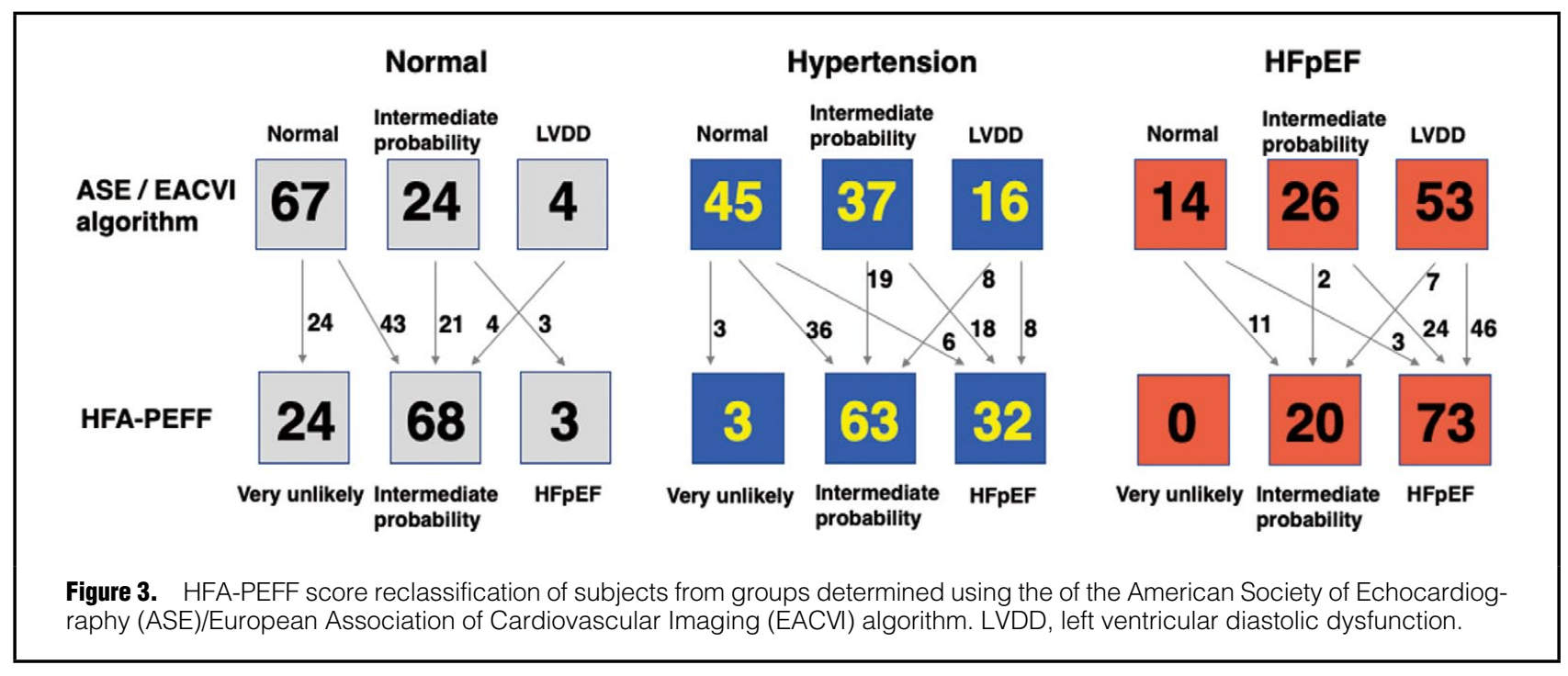


subjects and patients with preserved LVEF.,18-21 Although there have not been sufficient studies investigating the use of GLS for HFpEF, in this study we confirmed the ability of GLS to distinguish HFpEF. Unfortunately, satisfying the GLS criterion in this algorithm contributed little to the $\mathrm{HFpEF}$ diagnosis. Because GLS was able to discriminate between the 3 groups across all subjects (Table 2), if it is used as a first-line criterion, GLS may make a greater contribution to identifying HFpEF. Larger studies are needed to evaluate the value of GLS as a key component of the HFA-PEFF score in the assessment of HFpEF.

\section{Advantage of Comprehensive Evaluation by the HFA-PEFF Score}

The present study demonstrated the ability of the HFAPEFF scoring system to diagnose HFpEF. Just as the definition of HFpEF has historically depended on LV diastolic dysfunction, the results of the present study support the presence of advanced LV diastolic dysfunction determined by echocardiography as an important component of HFpEF. However, the key point of the HFAPEFF scoring system is that it also uses advanced morphological analysis and assessment of BNP concentrations to diagnose HFpEF in subjects without advanced LV diastolic dysfunction.

Another interesting finding of this study is that one-third of HT subjects, who had no prior HF events, were diagnosed with HFpEF based on the HFA-PEFF score. The prevalence of $\mathrm{HFpEF}$ in this study was larger than a previous validation study in which the number of control subjects was smaller and the subjects were younger than in the present study. ${ }^{13}$ The progression from HT to HF is well known, and the main pathophysiology is LV diastolic dysfunction rather than systolic dysfunction. ${ }^{22}$ In addition, patients with LV diastolic dysfunction but without a diagnosis of $\mathrm{HF}$ are common in the clinical setting. ${ }^{9}$ Interestingly, in the present study, three-quarters of reclassifications to $\mathrm{HFpEF}$ were observed in HT subjects without LV diastolic dysfunction according to the ASE/EACVI algorithm, suggesting the advantage of performing a comprehensive evaluation using the HFA-PEFF score. Therefore, a workup with the HFA-PEFF scoring system may contribute to a more precise diagnosis of early stage HFpEF in elderly patients with HT who have not been diagnosed with HF. Although stress tests or invasive hemodynamic evaluations may be needed to determine HF status, classification as $\mathrm{HFpEF}$ may be the first step in preventing the progression of HF status.

Finally, the scoring system often classified subjects in the healthy control and HT groups as being in the "intermediate probability" group. Previous studies have reported that hemodynamic assessment during exercises in patients with or suspected HFpEF is useful in diagnosing symptoms on exertion, elucidating the pathophysiological mechanism, and predicting the prognosis. ${ }^{23-26}$ The HFA-PEFF algorithm recommends that the population without a definite diagnosis proceeds to the next diagnostic step, such as exercise stress echocardiography or invasive hemodynamic measurements, which is an important point from the perspective of preventive medicine in subclinical cases.

\section{Study Limitations}

Because this study was a cross-sectional study, we could not evaluate the usefulness of the function tests in identifying $\mathrm{HFpEF}$ among a subclinical but undiagnosed $\mathrm{HFpEF}$ population; further longitudinal studies are needed to address this issue.

This study was limited to the late elderly, therefore another algorithm with an aging criterion was not assessed for comparison. ${ }^{26}$ Furthermore, we did not collect information about medications, so the effects of medications on the HFA-PEFF algorithm could not be assessed. Finally, subjects with a previous history of cardiovascular disease were excluded from this study, except for HFpEF patients. However, because the study was performed in an elderly population, it is possible that the subjects may have had occult coronary artery disease, which may have had an effect on diastolic function.

\section{Conclusions}

In the late elderly (aged $>75$ years), workup with echocardiography and BNP evaluations as part of the HFA-PEFF algorithm was able to identify subjects with HFpEF with high probability. In addition, most of the healthy control late elderly and subjects with HT were also classified as having HFpEF or having an intermediate probability of $\mathrm{HFpEF}$, suggesting that the HFA-PEFF scoring system could contribute to the detection of early stage HFpEF in the subclinical late elderly.

\section{Sources of Funding}

This study was supported by a grant from the Japanese Society of Echocardiography, as well as a grant from JSPS KAKENHI (Grant no. JP16K09417).

\section{Disclosures}

N.O. is the Associate Editor for Circulation Journal. The remaining authors have no conflicts of interest to declare.

\section{IRB Information}

This study was approved by the Ethics Committee of the University of Tsukuba (Reference no. H28-119).

\section{References}

1. United Nations, Department of Economic and Social Affairs Population Division. World population ageing 2019. https:// www.un.org/en/development/desa/population/publications/pdf/ ageing/WorldPopulationAgeing2019-Report.pdf (accessed May $8,2020)$.

2. Arai H, Ouchi Y, Toba K, Endo T, Shimokado K, Tsubota K, et al. Japan as the front-runner of super-aged societies: Perspectives from medicine and medical care in Japan. Geriatr Gerontol Int 2015; 15: 673-687.

3. Heidenreich PA, Albert NM, Allen LA, Bluemke DA, Butler J, Fonarow GC, et al. Forecasting the impact of heart failure in the United States: A policy statement from the American Heart Association. Circ Heart Fail 2013; 6: 606-619.

4. Ponikowski P, Anker SD, AlHabib KF, Cowie MR, Force TL, $\mathrm{Hu}$ S, et al. Heart failure: Preventing disease and death worldwide. ESC Heart Fail 2014; 1: 4-25.

5. Shah AM, Claggett B, Loehr LR, Chang PP, Matsushita K, Kitzman D, et al. Heart failure stages among older adults in the community: The Atherosclerosis Risk in Communities Study. Circulation 2017; 135: 224-240.

6. Westermann D, Kasner M, Steendijk P, Spillmann F, Riad A, Weitmann K, et al. Role of left ventricular stiffness in heart failure with normal ejection fraction. Circulation 2008; 117: 2051-2060.

7. Borlaug BA, Jaber WA, Ommen SR, Lam CS, Redfield MM, Nishimura RA. Diastolic relaxation and compliance reserve during dynamic exercise in heart failure with preserved ejection fraction. Heart 2011; 97: 964-969.

8. Cheng CP, Noda T, Nozawa T, Little WC. Effect of heart failure on the mechanism of exercise-induced augmentation of mitral valve flow. Circ Res 1993; 72: 795-806. 
9. Redfield MM, Jacobsen SJ, Burnett JC Jr, Mahoney DW, Bailey KR, Rodeheffer RJ. Burden of systolic and diastolic ventricular dysfunction in the community: Appreciating the scope of the heart failure epidemic. JAMA 2003; 289: 194-202.

10. Borlaug BA. Evaluation and management of heart failure with preserved ejection fraction. Nat Rev Cardiol 2020; 17: 559-573.

11. Shah AM, Shah SJ, Anand IS, Sweitzer NK, O'Meara E, Heitner $\mathrm{JF}$, et al. Cardiac structure and function in heart failure with preserved ejection fraction: Baseline findings from the echocardiographic study of the Treatment of Preserved Cardiac Function Heart Failure with an Aldosterone Antagonist Trial. Circ Heart Fail 2014; 7: 104-115.

12. Pieske B, Tschöpe C, de Boer RA, Fraser AG, Anker SD, Donal $\mathrm{E}$, et al. How to diagnose heart failure with preserved ejection fraction: The HFA-PEFF diagnostic algorithm: A consensus recommendation from the Heart Failure Association (HFA) of the European Society of Cardiology (ESC). Eur Heart J 2019; 40: $3297-3317$.

13. Barandiarán Aizpurua A, Sanders-van Wijk S, Brunner-La Rocca HP, Henkens M, Heymans S, Beussink-Nelson L, et al. Validation of the HFA-PEFF score for the diagnosis of heart failure with preserved ejection fraction. Eur J Heart Fail 2020; 22: $413-421$

14. Seo Y, Ishizu T, Ieda M, Ohte N; J-LONG Study Investigators. Elderly Japanese standard data of echocardiography; from J-LONG study. $J$ Echocardiogr 2020; 18: $175-182$.

15. Lang RM, Badano LP, Mor-Avi V, Afilalo J, Armstrong A, Ernande L, et al. Recommendations for cardiac chamber quantification by echocardiography in adults: An update from the American Society of Echocardiography and the European Association of Cardiovascular Imaging. J Am Soc Echocardiogr 2015; 28: $1-39$.

16. Nagueh SF, Smiseth OA, Appleton CP, Byrd BF III, Dokainish $\mathrm{H}$, Edvardsen T, et al. Recommendations for the evaluation of left ventricular diastolic function by echocardiography: An update from the American Society of Echocardiography and the European Association of Cardiovascular Imaging. Eur Heart $J$ Cardiovasc Imaging 2016; 17: $1321-1360$.

17. Ommen SR, Nishimura RA, Appleton CP, Miller FA, Oh JK, Redfield MM, et al. Clinical utility of Doppler echocardiography and tissue Doppler imaging in the estimation of left ventricular filling pressures: A comparative simultaneous Dopplercatheterization study. Circulation 2000; 102: 1788-1794.

18. Russo C, Jin Z, Elkind MS, Rundek T, Homma S, Sacco RL, et al. Prevalence and prognostic value of subclinical left ventricular systolic dysfunction by global longitudinal strain in a community-based cohort. Eur J Heart Fail 2014; 16: 1301-1309.

19. Biering-Sørensen T, Biering-Sørensen SR, Olsen FJ, Sengeløv M, Jørgensen PG, Mogelvang R, et al. Global longitudinal strain by echocardiography predicts long-term risk of cardiovascular morbidity and mortality in a low-risk general population: The Copenhagen City Heart Study. Circ Cardiovasc Imaging 2017; 10: $\mathrm{e} 005521$.

20. Ng ACT, Prihadi EA, Antoni ML, Bertini M, Ewe SH, Ajmone Marsan N, et al. Left ventricular global longitudinal strain is predictive of all-cause mortality independent of aortic stenosis severity and ejection fraction. Eur Heart J Cardiovasc Imaging 2018; 19: 859-867.

21. Saito M, Khan F, Stoklosa T, Iannaccone A, Negishi K, Marwick TH. Prognostic implications of LV strain risk score in asymptomatic patients with hypertensive heart disease. JACC Cardiovasc
Imaging 2016; 9: 911-921.

22. Levy D, Larson MG, Vasan RS, Kannel WB, Ho KK. The progression from hypertension to congestive heart failure. JAMA 1996; 275: $1557-1562$.

23. Borlaug BA, Nishimura RA, Sorajja P, Lam CS, Redfield MM. Exercise hemodynamics enhance diagnosis of early heart failure with preserved ejection fraction. Circ Heart Fail 2010; 3: $588-595$.

24. Dorfs S, Zeh W, Hochholzer W, Jander N, Kienzle RP, Pieske $\mathrm{B}$, et al. Pulmonary capillary wedge pressure during exercise and long-term mortality in patients with suspected heart failure with preserved ejection fraction. Eur Heart J 2014; 35: 3103-3112.

25. Reddy YNV, Obokata M, Wiley B, Koepp KE, Jorgenson CC, Egbe A, et al. The haemodynamic basis of lung congestion during exercise in heart failure with preserved ejection fraction. Eur Heart J 2019; 40: $3721-3730$.

26. Reddy YNV, Carter RE, Obokata M, Redfield MM, Borlaug BA. A simple, evidence-based approach to help guide diagnosis of heart failure with preserved ejection fraction. Circulation 2018; 138: $861-870$.

\section{Appendix}

The J-LONG Study investigators are as follows: Masako Asakawa (JR Tokyo General Hospital, Tokyo), Chikage Oshita (JR Hiroshima Hospital, Hiroshima), Masanori Takada (Kawasaki Hospital, Hyogo), Mitsufumi Furuya (Koyama Memorial Hospital, Ibaraki), Fumiko Sakamaki, Hitomi Hashimoto (Tsukuba Central Hospital, Ibaraki), Takako Minami (Nagasaki University Hospital, Nagasaki), Harutoshi Tamura (Yamagata University School of Medicine, Yamagata), Akiko Okunaga, Yuichi Oshima (Kinki Central Hospital, Hyogo), Yoshie Omori (Nikko Memorial Hospital, Hokkaido), Takuma Aoyama (Kizawa Memorial Hospital, Gifu), Takako Iino (Akita University Graduate School of Medicine, Akita), Makoto Saito (Kitaishikai Hospital, Ehime), Tetsuya Kasuga (Nanpeidai Medical Clinic, Ibaraki), Taro Minagawa (Minagawa Clinic, Gifu), Satoshi Kibira (Kibira Medical Clinic, Akita), Hidekazu Tanaka (Kobe University Graduate School of Medicine, Hyogo), Yoshihisa Horizoe (Ebino City Hospital, Miyazaki), Hiroyoshi Yamamoto, Shintaro Beppu (Osaka Minato Central Hospital), Misun Pak (Shimane University Faculty of Medicine, Shimane), Miyako Nagumo (National Hospital Organization Saitama Hospital, Saitama), Masaki Izumo (St. Marianna University School of Medicine, Kanagawa), Atsushi Kobayashi (Fukushima Medical University, Fukushima), Shohei Kikuchi (Nagoya City University Graduate School of Medical Sciences, Aichi), Noriaki Iwahashi (Yokohama City University Medical Center, Kanagawa), Kotaro Numaguchi (National Hospital Organization Kyushu Medical Center, Fukuoka), Toshinari Onishi (Osaka University Graduate School of Medicine, Osaka), Yuki Izumi (Nippon Medical School, Tokyo), Hirotsugu Yamada, Kenya Kusunose (Tokushima University Graduate School of Biomedical Sciences, Tokushima), Hideo Kawakami (Ehime Prefectural Imabari Hospital, Ehime), and Hiroyuki Iwano (Faculty of Medicine and Graduate School of Medicine, Hokkaido University, Hokkaido).

\section{Supplementary Files}

Please find supplementary file(s); http://dx.doi.org/10.1253/circj.CJ-20-0784 